\title{
Quenched phosphorescence as alternative detection mode in the chiral separation of methotrexate by electrokinetic chromatography
}

\author{
María Castro-Puyana • Ivonne Lammers • Joost Buijs • \\ Cees Gooijer • Freek Ariese
}

Received: 30 January 2011 /Revised: 28 March 2011 /Accepted: 6 April 2011 /Published online: 18 April 2011

(C) The Author(s) 2011. This article is published with open access at Springerlink.com

\begin{abstract}
Quenched phosphorescence was used, for the first time, as detection mode in the chiral separation of methotrexate (MTX) enantiomers by electrokinetic chromatography. The detection is based on dynamic quenching of the strong emission of the phosphorophore 1-bromo-4naphthalene sulfonic acid (BrNS) by MTX under deoxygenated conditions. The use of a background electrolyte with $3 \mathrm{mg} / \mathrm{mL}$ 2-hydroxypropyl- $\beta$-cyclodextrin and $20 \%$ $\mathrm{MeOH}$ in $25 \mathrm{mM}$ phosphate buffer (pH 7.0) and an applied voltage of $30 \mathrm{kV}$ allowed the separation of L-MTX and its enantiomeric impurity D-MTX with sufficient resolution. In the presence of $1 \mathrm{mM}$ BrNS, a detection limit of $3.2 \times$ $10^{-7} \mathrm{M}$ was achieved, about an order of magnitude better than published techniques based on UV absorption. The potential of the method was demonstrated with a degradation study and an enantiomeric purity assessment of LMTX. Furthermore, L-MTX was determined in a cell culture extract as a proof-of-principle experiment to show the applicability of the method to biological samples.
\end{abstract}

Keywords Capillary electrophoresis · Chiral analysis . Cyclodextrin $\cdot$ Enantiomeric purity

\section{Introduction}

L-Methotrexate (L-MTX, amethopterin, (2S)-2-[[4-[(2,4diaminopteridin-6-yl)methyl-methylamino]benzoyl]amino]

M. Castro-Puyana · I. Lammers · J. Buijs · C. Gooijer •

F. Ariese $(\triangle)$

Department of Biomolecular Analysis \& Spectroscopy,

LaserLaB, VU University Amsterdam,

De Boelelaan 1083,

1081 HV Amsterdam, the Netherlands

e-mail: f.ariese@few.vu.nl pentanedioic acid) (Fig. 1) is an antimetabolite of folic acid that is widely used for the treatment of several cancers, including childhood leukemia, head and neck cancer, and micrometastases of osteosarcoma. More recently, it is also being used at low dose for the treatment of rheumatoid arthritis, psoriasis, and steroid-dependent asthma [1-3]. L-MTX acts by inhibiting the enzyme dihydrofolate reductase (i.e., disruption of folate biosynthesis) due to its greater affinity for the enzyme than the closely related folic acid. This means that in the presence of L-MTX, key reactions in cell division are shut down [4]. The D-enantiomer (D-MTX) is subject to significantly different pharmacokinetics; it has a similar inhibitory effect on dihydrofolate reductase, but its antitumor effect is reduced $[1,5]$. For that reason, D-MTX has to be considered as a chiral impurity of L-MTX; it can originate from the synthesis of L-MTX or from racemization during the shelf life of the pharmaceutical product under improper storage conditions [6]. Regulatory requirements regarding the purity of chiral drugs have generally become stricter in recent years; therefore, the development of analytical methodologies to test for enantiomeric impurities in pharmaceutical formulations is of great importance.

Among the analytical techniques currently used for the purity control of pharmaceuticals, electrokinetic chromatography (EKC), a mode of capillary electrophoresis (CE), was shown to be a powerful tool in the field of chiral separations [7-17]. With UV absorption detection, which is still used most often in CE, the concentration sensitivity is limited by the optical path length of the capillary. For compounds with a chromophoric group, concentration limits in the micromolar range may be achieved in the most favorable cases [18]. Such detection limits may not be sufficiently low for biomedical samples or if low concentrations of enantiomeric impurities have to be detected. Therefore, we set out to investigate whether luminescence 


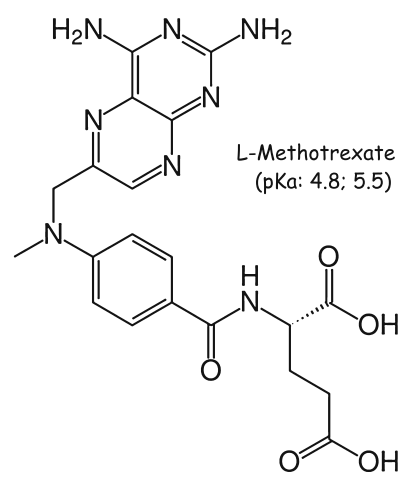

Fig. 1 Structure and $\mathrm{p} K_{\mathrm{a}}$ of L-methotrexate

methods (preferably without chemical derivatization) can provide a suitable alternative.

Next to fluorescence detection, which is well established in CE, room temperature phosphorescence (RTP) in liquid deoxygenated solutions was shown to be an attractive luminescence spectroscopic method for detection in CE [19-27]. Apart from direct phosphorescence, where the analyte is excited by light of an appropriate wavelength and phosphoresces itself, several indirect phosphorescence modes can be distinguished. In one sensitized mode, the analyte is excited and transfers its excitation energy to a more strongly phosphorescent acceptor added to the background electrolyte (BGE), after which the emission of the latter is observed. That approach was used in a previous paper on the enantioseparation and phosphorescence detection of bupropion [27]. Alternatively, one can excite a donor reagent with excellent absorption properties, which then transfers its electronic energy to the analyte of which the emission is detected [28]. Finally, in the quenched mode, a phosphorescent reagent is excited and its phosphorescence quantum yield is reduced by a biomolecular quenching process with an analyte molecule, which leads to negative peaks in the electropherogram $[20,21,29,30]$. Importantly, in the latter mode, also non-phosphorescent analytes can be detected, which is the case in this study. It should be noted that quenched phosphorescence is different from the indirect UV absorption mode, which is based on electrophoretic displacement of the BGE by the analytes.

Examination of the literature reveals that chiral discrimination of MTX has been achieved by means of different analytical techniques such as amperometric biosensors [31], voltammetric methods [32], potentiometric membrane electrodes [1], TLC [33], HPLC [6, 34, 35], and CE $[5,36-39]$. In the case of CE, the chiral separation of MTX and other compounds was carried out to evaluate vancomycin [36], D-(+)-tubocurarine [37], and cyclosophoraoses [39] as chiral selector. Moreover, Kuo et al. [38] developed a method for the enantiomeric analysis of
MTX in pharmaceuticals using 2-hydroxypropyl- $\beta$-cyclodextrin $(\mathrm{HP}-\beta-\mathrm{CD})$ as chiral selector. However, this method was only applied to verify the content of L-MTX in tablets and injection formulations, but not for purity control. On the other hand, Gotti et al. [5] carried out the determination of achiral and chiral impurities of L-MTX in pharmaceuticals using a micellar EKC system with $\beta-C D$.

In this work, we explore the applicability of phosphorescence as detection mode for the chiral separation of MTX by EKC. First, the direct and indirect phosphorescence properties of MTX were investigated in batch experiments. The quenched phosphorescence mode was found to be appropriate for the detection of MTX in combination with its chiral separation. In addition, the influence of different experimental conditions on the enantiomeric separation was also studied. Finally, the method developed in this work was applied to the enantiomeric purity control of L-MTX in a pharmaceutical formulation, to a short-term stability study, and to the determination of L-MTX in a cell culture extract.

\section{Material and methods}

Reagents and samples

All reagents employed were of analytical quality. Sodium phosphate monobasic, methanol, and $\beta-\mathrm{CD}$ were purchased from Sigma (St. Louis, MO, USA). Sodium hydroxide was obtained from Fluka (Buchs, Switzerland). ( \pm )-Camphorquinone (CQ) and HP- $\beta-C D$ were from Aldrich (Steinheim, Germany). 1-Bromo-4-naphthalenesulfonic acid (BrNS) was synthesized in-house [40]. Water used for preparing the solutions was purified through a Milli-Q system from Millipore (Bedford, MA, USA). L-MTX was obtained from Aldrich (Steinheim, Germany), and D-MTX was kindly provided by Dr. R. Gotti (University of Bologna, Italy). The pharmaceutical formulation analyzed (Emthexate PF, $100 \mathrm{mg} / \mathrm{mL}$; Pharmachemie BV, Haarlem, the Netherlands) and the L-MTX containing cell culture extract were kindly provided by Dr. G. Jansen and I. Kathman (VU Medical Center, Amsterdam, the Netherlands).

Instrumentation

A Cary Eclipse Luminescence Spectrometer (Varian, Melbourne, Australia) equipped with a pulsed Xe lamp was used for the batch experiments. The samples were deoxygenated using a stable nitrogen flow in a long-necked cuvette with a stopper and narrow vent (Hellma, Müllheim, Germany). The temperature was kept at $20{ }^{\circ} \mathrm{C}$ using the single-cell Peltier Cooler of the spectrometer. The phosphorescent reagents BrNS and CQ were excited at 294 or 
$470 \mathrm{~nm}$, respectively. Excitation and emission bandwidths were set to $10 \mathrm{~nm}$ and the PMT voltage was $600 \mathrm{~V}$. To avoid detecting interfering scatter and fluorescence, a delay time of $0.1 \mathrm{~ms}$ and a gate time of $5.0 \mathrm{~ms}$ were used. For the lifetime measurements, the initial delay time and the gating time were set at $0.05 \mathrm{~ms}$. Decay curves were fitted in OriginPro 8.

EKC separations were carried out using a CE system (200 series) from Prince Technologies (Emmen, the Netherlands) with an uncoated fused silica capillary of 75- $\mu \mathrm{m}$ i.d. (375- $\mu \mathrm{m}$ o.d.) and an effective length of $70 \mathrm{~cm}$ (total length $120 \mathrm{~cm}$ ) from Polymicro (Phoenix, AZ, USA). The measurements were made at room temperature, the voltage applied was $30 \mathrm{kV}$, and the samples were injected by pressure $(50 \mathrm{mbar}$ for $0.08 \mathrm{~min})$. Since deoxygenation is required to observe RTP, a modified vial was used to purge the buffer solution continuously with nitrogen [19]. The nitrogen flow rate was kept constant with a flow regulator. Before first use, a new capillary was rinsed with $1 \mathrm{M} \mathrm{NaOH}$ for $30 \mathrm{~min}$, followed by $2 \mathrm{~min}$ with Milli-Q water, $60 \mathrm{~min}$ with the buffer, and $5 \mathrm{~min}$ with the BGE (purged with nitrogen for at least $10 \mathrm{~min}$ before the start of the analysis and during the separation to remove oxygen). Between runs, the capillary was rinsed with BGE for $2 \mathrm{~min}$.

An LS-40 Luminescence LC detector (Perkin-Elmer, Beaconsfield, U.K.), adapted for CE [30], was used as phosphorescence detection system. The delay time was $0.08 \mathrm{~ms}$ and the gating time $3.00 \mathrm{~ms}$. Lamp excitation was performed at $294 \mathrm{~nm}$ and emission was collected by means of the total emission mirror and the 390-nm cutoff filter provided with the instrument.

\section{Analytical procedure}

Running buffers were prepared by dissolving the appropriate amount of sodium phosphate in Milli-Q water, adjusting the $\mathrm{pH}$ with $1 \mathrm{M} \mathrm{NaOH}$ before completing the volume to get the desired buffer concentration $(25 \mathrm{mM})$. BGEs were prepared by dissolving the appropriate amount of cyclodextrins in the buffer solution. Stock solutions of $0.025 \mathrm{M} \mathrm{BrNS}$ and $0.1 \mathrm{M}( \pm)-\mathrm{CQ}$ were prepared in water and $\mathrm{MeOH}$, respectively, and stored at $5{ }^{\circ} \mathrm{C}$. Stock solutions of L- or D-MTX were prepared by dissolving the appropriate amount of each enantiomer in $0.1 \mathrm{M} \mathrm{NaOH}$ up to a final concentration of $10^{-3} \mathrm{M}$ (stored at $-20^{\circ} \mathrm{C}$ ). Aliquots were diluted in water to get solutions with the desired concentrations (using an L/D ratio of $2: 1$ for easy distinction of the two peaks).

Human CEM leukemia cells $\left(5 \times 10^{6}\right.$ CEM-WT) were centrifuged $(528 \times \mathrm{g}$ for $5 \mathrm{~min}$ at room temperature), and the pellet was washed with $20 \mathrm{~mL}\left(4^{\circ} \mathrm{C}\right)$ Hanks balanced salt solution (HBSS) buffer containing $20 \mathrm{mM}$ HEPES,
$140 \mathrm{mM} \mathrm{NaCl}, 6 \mathrm{mM} \mathrm{KCl}, 2 \mathrm{mM} \mathrm{MgCl}_{2}$, and $6 \mathrm{mM}$ glucose, $\mathrm{pH} 7.4$, set with $\mathrm{NaOH}$. After centrifugation $\left(528 \times \mathrm{g}\right.$ for $5 \mathrm{~min}$ at $\left.4{ }^{\circ} \mathrm{C}\right)$, the pellet was resuspended in $1 \mathrm{~mL}$ HBSS $\left(4{ }^{\circ} \mathrm{C}\right)$ followed by centrifugation $(15,294 \times g$ for $5 \mathrm{~min}$ at $4{ }^{\circ} \mathrm{C}$ ). The pellet obtained was resuspended in $150 \mu \mathrm{L}$ HBSS $\left(4{ }^{\circ} \mathrm{C}\right)$ and spiked with $1 \mu \mathrm{M}$ MTX and $40 \mu \mathrm{L}$ ice-cold $40 \%$ trichloroacetic acid. After $20 \mathrm{~min}$, the cell suspension was shaken and centrifuged at $15,294 \times \mathrm{g}$ for 5 min at $4{ }^{\circ} \mathrm{C}$, and the supernatant was separated and suspended in alamine/freon. Finally, the aqueous layer was separated by centrifugation $\left(15,294 \times g, 5 \mathrm{~min}, 4{ }^{\circ} \mathrm{C}\right)$ and stored at $-20{ }^{\circ} \mathrm{C}$.

All BGEs, standards, and samples were filtered through 0.2- $\mu \mathrm{m}$ syringe filters (Whatman, Dassel, Germany) before their use in the CE system.

\section{Results and discussion}

\section{Batch experiments}

According to the literature, the enantiomeric separation of MTX by EKC can be achieved using HP- $\beta-C D$ as chiral selector in phosphate buffer at $\mathrm{pH} 7.0$ [38]. Keeping in mind that the objective of this work was to investigate the feasibility of phosphorescence detection in $\mathrm{CE}$ as an alternative to UV detection, we employed these preliminary conditions to study the direct and indirect phosphorescence of MTX in batch.

In order to find optimum excitation conditions, first of all, the absorption and fluorescence spectra were recorded. In UV-Vis absorption, four maxima can be discerned, i.e., at 220, 259, 303, and $373 \mathrm{~nm}$. Under the experimental conditions, MTX was found to be weakly fluorescent, too weak to be of interest from an analytical detection point of view. Interestingly, in fluorescence instead of a single emission spectrum-as generally expected - two weak emission bands were found, i.e., at 356 and $465 \mathrm{~nm}$ (not shown). This is not due to the presence of two species, but is attributed to the fact that MTX is composed of two separate chromophoric moieties, connected by an aliphatic $-\mathrm{C}-\mathrm{N}-$ group. The corresponding excitation spectra are distinctly different: The maxima at 220 and $303 \mathrm{~nm}$ are associated with the 365-nm band; those at 259 and $373 \mathrm{~nm}$ with the 456-nm band. Thus, there are two suitable excitation wavelengths for MTX: 303 and $373 \mathrm{~nm}$, corresponding presumably with the benzoyl and the pteridin unit.

Also, two phosphorescence emission signals could be expected, but under deoxygenated conditions, no direct phosphorescence was observed at either excitation wavelength (303 and $373 \mathrm{~nm}$ ). Therefore, indirect phosphorescence in sensitized and quenched mode was explored. 
For this purpose, the phosphorophores BrNS and CQ were tested; both compounds have been used extensively in earlier studies $[19,21-23,26,28,40,41]$. In the sensitized phosphorescence mode, MTX, is excited and transfers its excitation energy to BrNS or CQ, of which the phosphorescence is then observed. When BrNS was used as acceptor, MTX was only excited at $373 \mathrm{~nm}$ since $303 \mathrm{~nm}$ is too close to the maximum excitation wavelength of BrNS (294 $\mathrm{nm}$ ) and would thus result in a high background from direct excited acceptor. In the case of BrNS, sensitized phosphorescence was not observed. For $\mathrm{CQ}$, the energy transfer from MTX (excited at 303 or $373 \mathrm{~nm}$ ) resulted in only a small increase in the CQ phosphorescence signal (data not shown). Thus, we concluded that also sensitized phosphorescence is not a promising option for MTX detection. Presumably, for both chromophoric moieties, the triplet state energies are too low for efficient transfer to BrNS or CQ.

In the dynamically quenched mode, BrNS or CQ are excited (at 294 or $470 \mathrm{~nm}$, respectively), and their phosphorescence intensity is monitored. If MTX has a lower triplet state energy, the transfer of excitation energy from the phosphorophore to MTX can take place, which diminishes the observed phosphorescence intensity from $I_{0}$ to $I$ according to the Stern-Volmer equation:

$I_{0} / I=1+k_{\mathrm{q}}[Q] \tau_{0}$

where $k_{\mathrm{q}}$ is the quenching rate constant, $[Q]$ the analyte concentration, and $\tau_{0}$ the triplet lifetime of the phosphorophore in the absence of quenching analyte [29, 30].

Figure 2 shows the phosphorescence emission spectra of BrNS and CQ, recorded in batch in the presence of HP$\beta-C D$, with and without $10^{-5} \mathrm{M}$ L-MTX. In the absence of quencher, the phosphorescence intensity of BrNS was about 50 times higher than that of CQ. For both phosphorophores, the presence of L-MTX resulted in a decrease of the signal: $I_{0} / I=15.3$ for BrNS and 1.5 for CQ. Lifetime measurements showed that in both cases, we are indeed dealing with dynamic quenching: For instance, for BrNS, the phosphorescence lifetime decreased from 2.05 to $0.150 \mathrm{~ms}$ in the presence of MTX. This 14-fold decrease is very close to the observed change in intensity. The rate constant of quenching $k_{\mathrm{q}}$ was calculated from Eq. 1 as $7 \times 10^{8} \mathrm{M}^{-1} \mathrm{~s}^{-1}$, close to the typical rate constant of collisional quenching $\left(1-5 \times 10^{9} \mathrm{M}^{-1} \mathrm{~s}^{-1}\right)$.

In dynamically quenched phosphorescence detection for $\mathrm{CE}$, a high background signal (with relatively low noise) and a long triplet lifetime $\tau_{0}$ are preferred for maximum sensitivity. Therefore, the quenched mode with BrNS was selected for further experiments.
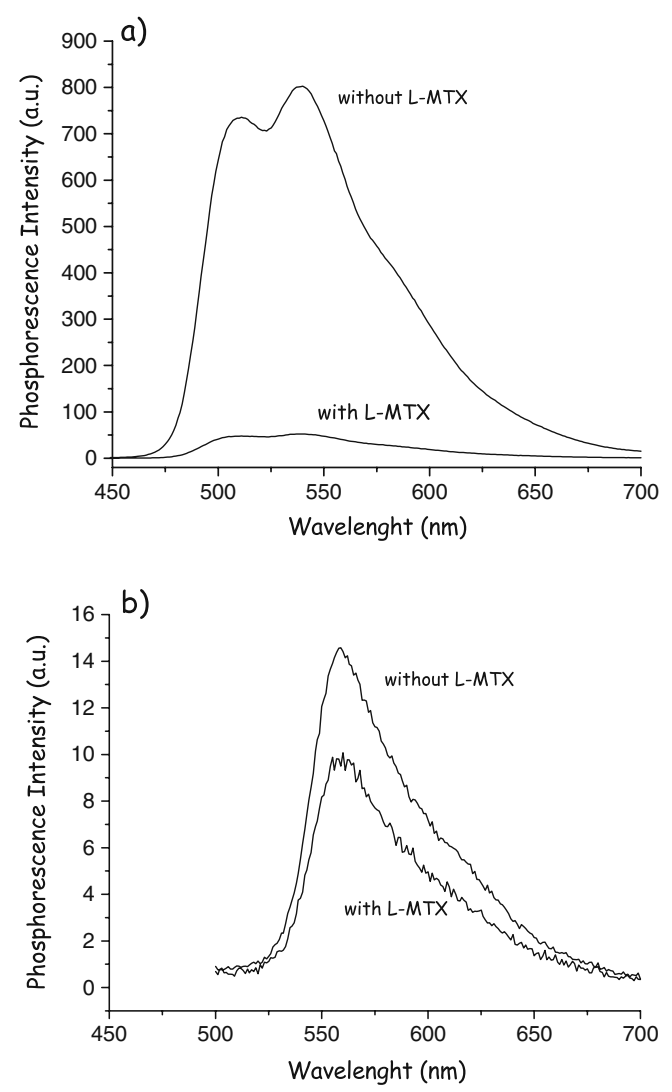

Fig. 2 Phosphorescence emission spectra of $10^{-5} \mathrm{M} \operatorname{BrNS}$ (a) and $2 \mathrm{mM}( \pm)$-CQ (b) with and without quenching by L-MTX $\left(10^{-5} \mathrm{M}\right)$ in $25 \mathrm{mM}$ phosphate buffer ( $\mathrm{pH}$ 7.0) with $3 \mathrm{mg} / \mathrm{mL}$ HP- $\beta-\mathrm{CD}$. Batch measurements: spectrometer settings - delay time, $0.1 \mathrm{~ms}$; gating time, $5.0 \mathrm{~ms} ; \lambda_{\text {exc }}=294 \mathrm{~nm}$ for BrNS and $470 \mathrm{~nm}$ for $( \pm)-C Q$; excitation and emission band widths, $10 \mathrm{~nm}$. All samples were purged with nitrogen to remove oxygen

\section{CE phosphorescence}

For the optimization of the CE separation, we started out from the experimental conditions described by Kuo et al. [38] with small modifications, such as the use of a longer capillary (at least $60 \mathrm{~cm}$ to the detection window instead of $30 \mathrm{~cm}$ since short capillary lengths are not practical with the LS40 detector), a higher CE voltage $(30 \mathrm{kV})$, and the presence of $1 \mathrm{mM}$ BrNS in the BGE. At first, only a partial resolution of MTX enantiomers was obtained. Therefore, various parameters such as $\mathrm{CD}$ concentration, methanol $(\mathrm{MeOH})$ percentage, and capillary length were studied in order to improve the chiral separation. These aspects were optimized as separate parameters.

The influence of the concentration of HP- $\beta-C D$ on the enantioseparation of MTX was investigated in the range from 1 to $10 \mathrm{mg} / \mathrm{mL}$ because the degree of complexation of the enantiomers is affected by the concentration of the chiral selector. The resolution gradually deteriorated when the concentration increased up to $10 \mathrm{mg} / \mathrm{mL}$. In addition, an 


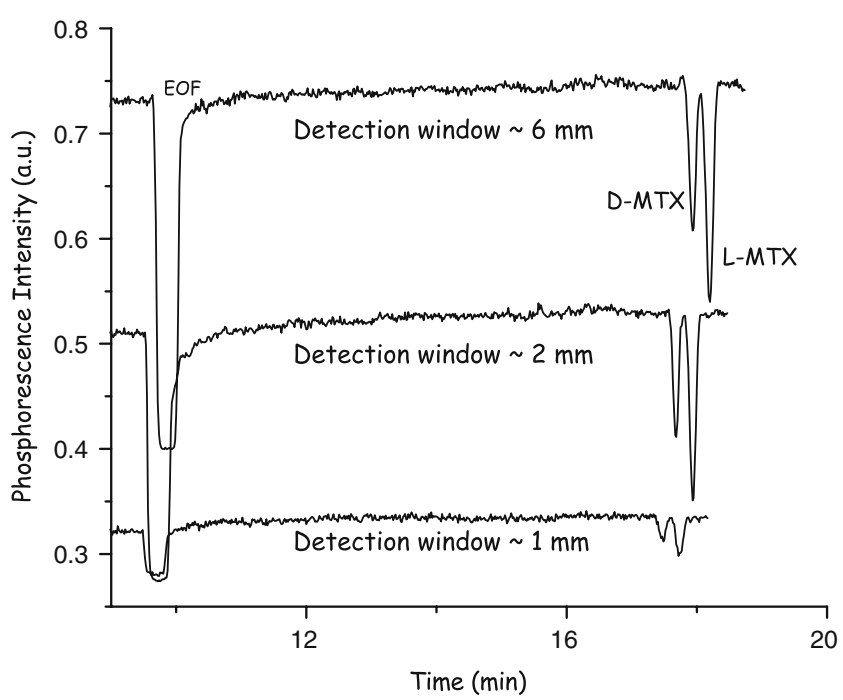

Fig. 3 EKC phosphorescence: influence of the detection window length on the phosphorescence intensity and chiral resolution of D,LMTX. Sample, $5 \times 10^{-6} \mathrm{M}$ L-MTX $+2.5 \times 10^{-6} \mathrm{M}$ D-MTX; BGE, $1 \mathrm{mM}$ BrNS with $3 \mathrm{mg} / \mathrm{mL} \mathrm{HP}-\beta-\mathrm{CD}$ and $20 \% \mathrm{MeOH}$ in $25 \mathrm{mM}$ phosphate $(\mathrm{pH} 7.0)$; untreated fused-silica capillary, $110 \mathrm{~cm}(60 \mathrm{~cm}$ to the detector window $) \times 75-\mu \mathrm{m}$ i.d. Other conditions are described in the experimental section. The BGE was purged with nitrogen to remove oxygen. The electropherograms are vertically shifted for clarity

increase in the $\mathrm{CD}$ concentration resulted in shorter migration times. Because of the lower electrophoretic mobility of the inclusion MTX/HP- $\beta-\mathrm{CD}$ complexes in comparison with the free analyte, the complexes migrate closer to the EOF. Eventually, $3 \mathrm{mg} / \mathrm{mL}$ of HP- $\beta-\mathrm{CD}$ was chosen for further experiments as a compromise between resolution and analysis time. Interestingly, the peak areas observed with quenched phosphorescence detection were hardly affected by the HP- $\beta-C D$ concentration, indicating that free and complexed MTX have similar quenching properties toward BrNS.

Since the CD concentration could not improve the separation further, the addition of different percentages of $\mathrm{MeOH}(10 \%, 20 \%$, and 25\%) was studied. Adding organic modifier to the running buffer may affect the enantiomeric resolution, as well as other parameters such as the EOF, the conductivity of the BGE, and the analysis time. With higher percentages of $\mathrm{MeOH}$, the chiral resolution improved, but this added to the analysis time. Twenty percent $\mathrm{MeOH}$ was chosen as a trade-off between both parameters. However, the enantioseparation of MTX was still only partial. We hypothesized that the 6-mm detection window of the lampbased phosphorescence detector could contribute to the overall band broadening. The detection window was reduced in size by means of black shrink tubing around the capillary. Figure 3 shows the electropherograms obtained for a solution of $5 \times 10^{-6} \mathrm{M}$ L-MTX and $2.5 \times$ $10^{-6} \mathrm{M}$ D-MTX using different detection window lengths.

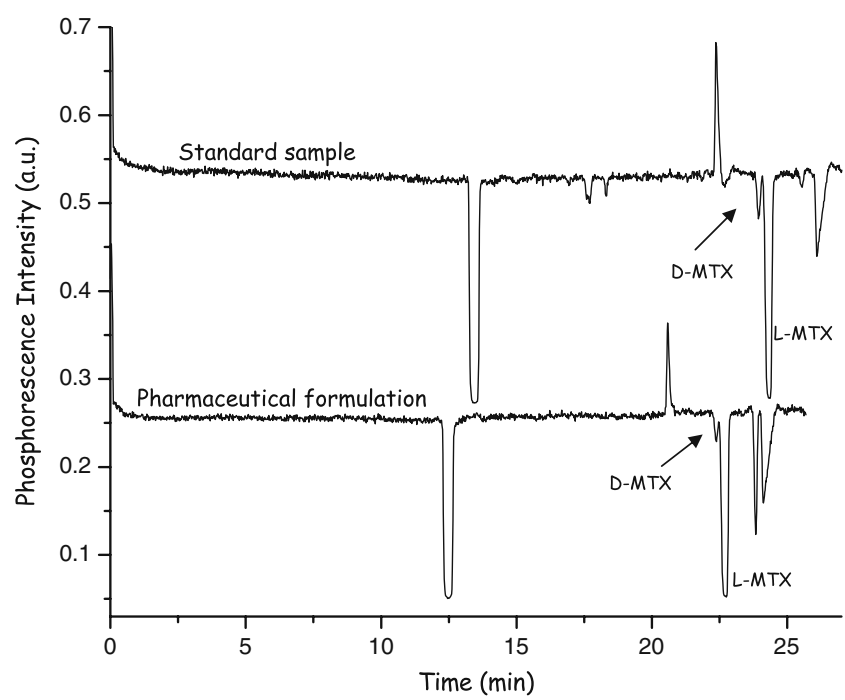

Fig. 4 D-MTX impurity determination in a standard sample (top) and in a pharmaceutical formulation (bottom); unspiked samples with a nominal L-MTX concentration of $1 \times 10^{-4} \mathrm{M}$. Untreated fused silica capillary, $120 \mathrm{~cm}(70 \mathrm{~cm}$ to the detector window $) \times 75-\mu \mathrm{m}$ i.d. Other conditions as in Fig. 3. The electropherograms are vertically shifted for clarity

Reducing the window to about $2 \mathrm{~mm}$ improved the resolution, but with even narrower detection windows, a significant loss of signal $\left(I_{0} / I\right)$ was observed. As a compromise, the 2-mm window was chosen for further experiments. The capillary length was increased (from 60 to $70 \mathrm{~cm}$ ) to further improve the separation. Finally, in order to find the optimal BrNS concentration and obtain the highest phosphorescence intensity, it was varied from 0.5 to $1.5 \mathrm{mM}$, but no major differences were observed. Since the use of $1 \mathrm{mM}$ BrNS resulted in a slightly higher phosphorescence intensity, it was chosen as the best BrNS concentration (data not shown).

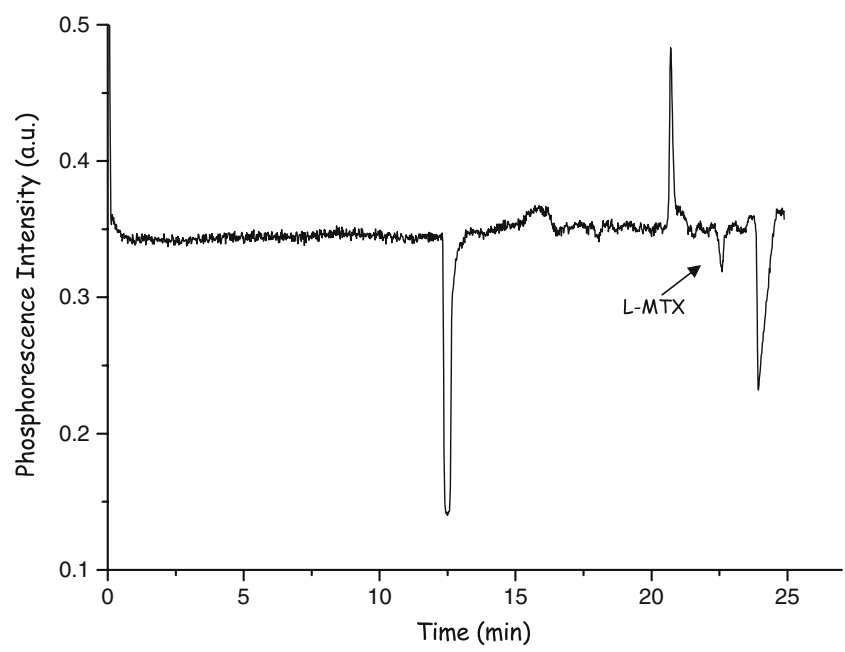

Fig. 5 EKC phosphorescence of L-MTX $(1 \mu \mathrm{M})$ in a cell culture extract. Experimental conditions as in Fig. 4 
In summary, the chiral separation of MTX enantiomers with quenched phosphorescence detection was achieved using as BGE $1 \mathrm{mM}$ BrNS with $3 \mathrm{mg} / \mathrm{mL}$ HP- $\beta-\mathrm{CD}$ and $20 \% \mathrm{MeOH}$ in $25 \mathrm{mM}$ phosphate buffer $(\mathrm{pH} 7.0)$ in a capillary of $70 \mathrm{~cm}$ to the detector (with a detection window of about $2 \mathrm{~mm}$ ). These conditions allowed the separation of MTX with a resolution of $\sim 1.5$. Finally, the LOD and LOQ were calculated from the $\mathrm{S} / \mathrm{N}$ ratio equal to 3 and 10 , respectively, when injecting a solution of $2.5 \times 10^{-6} \mathrm{M} \mathrm{L}$ MTX and $1.25 \times 10^{-6} \mathrm{M}$ D-MTX. A LOD of $3.2 \times 10^{-7} \mathrm{M}$ and a LOQ of $1.1 \times 10^{-6} \mathrm{M}$ were achieved for both enantiomers. This is a major improvement compared with the LODs reported in the literature for the chiral separation of MTX with UV absorption detection: $4 \times 10^{-6} \mathrm{M}$ for CE [38], $1.5 \times 10^{-6} \mathrm{M}$ for MECK [5], and $2 \times 10^{-6} \mathrm{M}$ for HPLC [6].

Application to formulations and biological samples

The developed method was applied to determine the optical purity of standards and a pharmaceutical formulation of LMTX. The level of D-MTX in both samples was quantified by means of the standard addition method. The percentages of spiked D-MTX ranged from $0 \%$ to $3 \%(w / w)$, relative to a nominal concentration of L-MTX of $10^{-4} \mathrm{M}$. The actual level of the L-enantiomer was too high to be determined immediately (upper limit of the calibration range is $1 \times$ $10^{-5} \mathrm{M}$ ) and was therefore verified separately after dilution.

In a quenching experiment, the peak heights do not increase linearly with the analyte concentration. Linear calibration curves were obtained by plotting $I_{0} / I$, according to the Stern-Volmer equation (Eq. 1), versus the percentage of D-MTX (X) added to the sample. The equations obtained, by means of a linear regression analysis, were: $y=0.228 x+1.248\left(r^{2}=0.9994\right)$ for the standard and $y=$ $0.249 x+1.148\left(r^{2}=0.9899\right)$ for the pharmaceutical formulation. Figure 4 shows the electropherograms obtained for both samples when $0 \%$ D-MTX was added. Percentages of $1.1 \%$ (chemical standard) and $0.6 \%$ D-MTX (pharmaceutical formulation) were calculated by solving the above equations for $y=1$.

A short-term degradation study was also carried out to investigate the possible racemization of L-MTX. Nine standard samples of $10^{-4} \mathrm{M}$ L-MTX were stored at 4, 20, and $37^{\circ} \mathrm{C}$ and analyzed after 2,6 , and $24 \mathrm{~h}$. The D-MTX levels were very similar in all samples (the ratios $I_{0} / I$ ranged from 1.30 to 1.36 ), which showed that no significant racemization of L-MTX occurred under the conditions studied.

Finally, as a proof-of-principle experiment for the application of the developed method to biological samples, L-MTX was determined in a cell culture extract from human CEM leukemia cells. The extract was spiked with $1 \mu \mathrm{M}$ L-MTX, a realistic level in pharmacokinetic and pharmacodynamic experiments. As demonstrated in Fig. 5, the intracellular components caused little interference in the quenched phosphorescence detection of L-MTX. The L-MTX peak was above the LOD level of $3.1 \times 10^{-7} \mathrm{M}$ (based on the noise level measured over the range 5-10 $\mathrm{min}$ ), but for proper quantitation, slightly higher levels or sample preconcentration would be preferred. The developed method will be useful for determining the intracellular concentration of MTX and can be applied in future studies on the dynamic equilibrium between influx and efflux routes.

\section{Concluding remarks}

Following the batch study of the phosphorescence characteristics of MTX (direct and indirect phosphorescence), the quenched phosphorescence mode was chosen as the detection method for the chiral separation of MTX enantiomers by EKC. Under the optimized conditions, $1 \mathrm{mM} \mathrm{BrNS}$ with $3 \mathrm{mg} / \mathrm{mL} \mathrm{HP}-\beta-\mathrm{CD}$ and $20 \% \mathrm{MeOH}$ in $25 \mathrm{mM}$ phosphate buffer (pH 7.0), the enantiomeric separation of L- and D-MTX was obtained with a resolution $\sim 1.5$. Under these conditions, a LOD of $3.2 \times$ $10^{-7} \mathrm{M}$ was achieved for both enantiomers, improving the LODs previously reported in the literature with about an order of magnitude. Finally, the developed method was satisfactorily applied to the enantiomeric purity control of standards and a pharmaceutical formulation. A short-term stability study did not show any major degree of racemization in aqueous solution up to $24 \mathrm{~h}$, not even at $37{ }^{\circ} \mathrm{C}$. The method was also found to be suitable for studying MTX in cell culture extracts.

Acknowledgments M.C.-P. thanks the Ministry of Science and Innovation (Spain) for her postdoctoral fellowship (Movilidad RRHH del Plan Nacional I-D+I 2008-0288). I.L. thanks the Dutch Foundation for Advancement of Science (NWO-CW) for financial support (ECHO grant no. 700.55.014). The authors also thank Dr. R. Gotti for the kind gift of D-MTX and Dr. G. Jansen and I. Kathman (VU Medical Center) for the pharmaceutical formulation and the preparation of the cell culture extract.

Open Access This article is distributed under the terms of the Creative Commons Attribution Noncommercial License which permits any noncommercial use, distribution, and reproduction in any medium, provided the original author(s) and source are credited.

\section{References}

1. Rat'ko AA, Stefan RI, van Staden JF, Aboul-Enein HY (2004) Talanta 64:145-150

2. Rodríquez Flores J, Castañeda Peñalvo G, Espinosa Mansilla A, Rodríguez Gómez MJ (2005) J Chromatogr B 819:141-147 
3. Li H, Luo W, Zeng Q, Lin Z, Luo H, Zhang Y (2007) J Chromatogr B 845:164-168

4. Roach MC, Gozel P, Zare RN (1988) J Chromatogr 426:129-140

5. Gotti R, El-Hady DA, Andrisano V, Bertucci C, El-Maali NA, Cavrini V (2004) Electrophoresis 25:2830-2837

6. El-Hady DA, El-Maali NA, Gotti R, Bertucci C, Mancini F, Andrisano V (2005) J Pharm Biomed Anal 37:919-925

7. Chankvetadze B (1997) Capillary electrophoresis in chiral analysis. Wiley, Chichester

8. Tanaka Y, Yanagawa M, Terabe S (1996) J High Resol Chromatogr 19:421-433

9. Fanali S (2000) J Chromatogr A 875:89-122

10. García-Ruiz C, Marina ML (2005) In: Marina ML, Rios A, Valcarcel M (eds) Analysis and detection in capillary electrophoresis, chapter 13. Elsevier, Amsterdam

11. Van Eeckhaut A, Michote Y (2006) Electrophoresis 27:28802895

12. Gübitz G, Schmid MG (2007) Electrophoresis 28:114-126

13. Chankvetadze B (2007) J Chromatogr A 1168:45-70

14. Ward TJ, Baker BA (2008) Anal Chem 80:4363-4372

15. Gübitz G, Schmid MG (2008) J Chromatogr A 1024:140-156

16. Fanali S (2009) Electrophoresis 30:S203-S210

17. Chankvetadze B (2009) Electrophoresis 30:S211-S221

18. Crego AL, Marina ML (2005) In: Marina ML, Rios A, Valcarcel $M$ (eds) Analysis and detection in capillary electrophoresis, chapter 5. Elsevier, Amsterdam

19. Kuijt J, Arraez Roman D, Ariese F, Brinkman UAT, Gooijer C (2002) Anal Chem 74:5139-5145

20. Kuijt J, Ariese F, Brinkman UAT, Gooijer C (2003) Anal Chim Acta 488:135-177

21. García-Ruiz C, Siderius M, Ariese F, Gooijer C (2004) Anal Chem 76:399-403

22. García-Ruiz C, Hu X, Ariese F, Gooijer C (2005) Talanta 66:634640
23. García Ruiz C, Scholtes MJ, Ariese F, Gooijer C (2005) Talanta 66:641-645

24. Zhang WH, Wang Y, Jin WJ (2007) Talanta 73:938-942

25. Zhang WH, Wang Y, Jin WJ (2008) Anal Chim Acta 622:157162

26. Lammers I, Buijs J, van der Zwan G, Ariese F, Gooijer C (2009) Anal Chem 81:6226-6233

27. Castro-Puyana M, Lammers I, Buijs J, Gooijer C, Ariese F (2010) Electrophoresis 31:3928-3936

28. Lammers I, Buijs J, Ariese F, Gooijer C (2010) Anal Chem 82:9410-9417

29. Kuijt J, Ariese F, Gooijer C (2005) In: Marina ML, Rios A, Valcarcel M (eds) Analysis and detection in capillary electrophoresis, chapter 7. Elsevier, Amsterdam

30. Kuijt J, Brinkman UAT, Gooijer C (1999) Anal Chem 71:13841390

31. Stefan RI, Bokretsion RG, van Staden JF, Aboul-Enein HY (2003) Talanta 60:983-990

32. El-Hady AD, Seliem MM, Gotti R, El-Maali NA (2006) Sens Actuators, B 113:978-988

33. Subert J, Slais K (2001) Pharmazie 56:355-360

34. Cramer SM, Schornagel JH, Kalghatgi KK, Bertino JR, Horváth C (1984) Cancer Res 44:1843-1846

35. Brandsteterova E, Marcincinova K, Lehotay J, Zbojova A, Halko J (1993) Neoplasma 40:241-245

36. Armstrong DW, Rundlett KL, Chen JR (1994) Chirality 6:496-509

37. Nair UB, Armstrong DW, Hinze WL (1998) Anal Chem 70:10591065

38. Kuo CY, Wu HL, Wu SM (2002) Anal Chim Acta 471:211-217

39. Lee S, Jung S (2003) Carbohydr Res 338:1143-1146

40. Kuijt J, van Teylingen R, Nijbacker T, Ariese F, Brinkman UAT, Gooijer C (2001) Anal Chem 73:5026-5029

41. Kuijt J, Ariese F, Brinkman UAT, Gooijer C (2003) Electrophoresis 24:1193-1199 